aufrüttelndes Plädoyer für eine glückliche Kindheit zu schreiben. Es ist zu hoffen, dass Eltern, Lehrer und Erzieher aber auch Kinder- und Jugendärzte motiviert werden, eine vorschnelle Pathologisierung von Entwicklungsverzögerungen zu vermeiden. Bis auf des Autors Meinungen über ADHS halte ich es für Eltern, aber auch für Kinder- ärzte als ein empfehlenswertes Buch, was sich auch nach einem anstrengendem Arbeitstag noch gut lesen lässt.

Dr. Kirsten Stollhoff

\title{
Begeisterung für die Schmerztherapie
}

_ Prof. Dr. Boris Zernikow ist Chefarzt des Deutschen Kinderschmerzzentrums in Datteln, dem Referenzzentrum in der Versorgung chronisch schmerzkranker Kinder und Jugendlicher. Viele der Koautoren stammen aus dem erfahrenen Team. Sehr gut verständlich und fesselnd geschrieben richten sich die Autoren an alle, die im medizinischen Bereich mit Kindern betraut sind. Der Text wird vereinzelt durch Tabellen und Abbildungen aufgelockert, bei durchgängig klar strukturiertem und übersichtlichem Aufbau. Der erste Teil beinhaltet sechs Kapitel: Was ist Schmerz?, Pathophysiologie, nozizeptives System von Früh- und Neugeborenen, Differenzialdiagnose der Schmerzursachen, Epidemiologie funktioneller Schmerzen sowie Messen und Erfassen von Schmerz. Es bietet praxisnahe Hinweise mit Angaben, wo die entsprechenden Fragebögen im Netz verfügbar sind. Im zweiten Teil wird die medikamentöse Therapie (klinisch-pharmakologi- sche Grundlagen der Schmerztherapie, Regional- und Lokalanästhesie) mit den für die tägliche Praxis wichtigen Angaben dargestellt - gut geeignet um noch einmal kurz nachzuschlagen. Die psychologischen Aspekte im dritten Teil (psychologische Interventionen bei akuten bzw. chronischen Schmerzen) sind sehr gut nach Alter und Gesprächspartner gegliedert. Sie bieten hilfreiche Tipps im Umgang mit den Eltern, deren Rolle immer wieder hervorgehoben wird.

Auch der vierte Teil (Schmerzmanagement in der pädiatrischen Pflege und ergänzende, naturheilkundlich orientierte Methoden) ist lesenswert, da er viele Anleitungen enthält, die den Eltern als „Hausmittel“ mitgegeben werden können und in Rezeptform enthalten sind (mit Bestellmöglichkeiten der einzelnen Substanzen). Akupunktur und Homöopathie werden besprochen und auch hier ärztliche Alltagstipps weitergegeben. Im fünften Teil findet jeder Leser seinen Bereich: von Dreimonatskoliken bis zur palliativen Schmerztherapie. Der letzte Teil zieht ein Resümee über die in den letzten Jahren erreichten Fortschritte. Im Serviceteil finden sich Tabellen und Fragebögen.

Insgesamt ist es ein hervorragendes und umfassendes Lehrbuch, das getragen wird von der Begeisterung der Autoren für die Schmerztherapie. Es bietet dem Leser eine Fülle von Anregungen zur Umsetzung in die Praxis. Dieses Buch kann ich uneingeschränkt empfehlen! Dr. Caroline Maxton

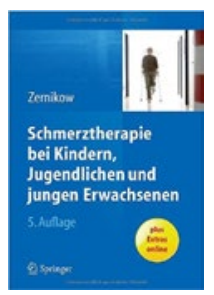

Boris Zernikow

Schmerztherapie bei Kindern, Jugendlichen und jungen Erwachsenen

Springer-Verlag, Berlin 2015 $79,99 €$ ISBN 978-3-662-45057-4

\section{Mitarbeiterführung: Es muss Spaß machen, bei Ihnen zu arbeiten!}

— Eine zeitgemäße Führungskultur ist eine wichtige Voraussetzung dafür, dass sich eine Praxis erfolgreich entwickelt. Das hängt unter anderem damit zusammen, dass in den meisten Arztpraxen die Ausgaben für Personal mit $40 \%$ oder mehr der größte Kostenblock sind. Aber auch die Zufriedenheit der Patienten ist gebunden an die Motivation des Praxisteams - seien dies MFA oder angestellte Ärzte. Den Zusammenhang zwischen diesen beiden Erfolgsfaktoren einer Praxis verdeutlichte der Fachanwalt für Arbeitsrecht Kai Höppner kürzlich beim Seminar „Personalführung und Mitarbeiterauswahl in der Praxis" in Braunschweig. Veranstalter waren die Deutsche Apotheker- und Ärztebank (apoBank) und das Institut für Wirtschaft und Praxis (IWP) in Kooperation mit der KV Niedersachsen. „Es muss Spaß machen, bei Ihnen zu arbeiten", so der Appell Höppners. Das komme auch bei den Patienten an, es spreche sich herum, es mache sich bemerkbar bei der Suche nach neuen Mitarbeitern.

Zu einer zeitgemäßen Führungskultur gehört laut Höppner vor allem die kooperative Zusammenarbeit mit den Mitarbeitern. Es sei wichtig, MFA und angestellten Ärzten Freiräume zu lassen, damit sie ihre Arbeit selbstständig machen und sich dabei wenigstens teilweise selbst entfalten können. Zu koppeln sei diese Freiheit aber immer mit konkreten Zielen, die dann in einem regelmäßig durchgeführten Gespräch zum Jahresende oder -beginn nachgehalten werden können. Solche „Kommunikationsrituale" wie Mitarbeitergespräche, so Höppner, seien auch wichtig, um festzustellen, wie zufrieden die Mitarbeiter mit ihrer Situation sind. Darüber hinaus seien regelmäßige Teamsitzungen in diesem Zusammenhang von Bedeutung. Dabei sind aktuelle Informationen zur Praxisentwicklung, etwa zur Erweiterung des Praxisspektrums, ebenso anzusprechen wie Fehler und Konflikte. Fehler sind immer als Chance zu sehen, aus ihnen zu lernen und damit zukünftige Fehler zu vermeiden. Nur so kann Offenheit erreicht werden und Fehler werden nicht unter den Teppich gekehrt. Zu den zehn Geboten einer zeitgemäßen Mitarbeiterführung gehöre auch, dass die Praxisinhaber bereit seien, ein kritisches Feedback ernst zu nehmen und darauf zu reagieren, sagte Höppner. Nicht zuletzt motiviere die Wertschätzung guter Arbeit von Mitarbeitern dazu, sich auch in Zukunft ins Zeug zu legen. Hauke Gerlof 